\title{
Epistemic democracy with defensible premises
}

Citation for published version (APA):

Dietrich, F. K., \& Spiekermann, K. (2010). Epistemic democracy with defensible premises. METEOR, Maastricht University School of Business and Economics. METEOR Research Memorandum No. 066 https://doi.org/10.26481/umamet.2010066

Document status and date:

Published: 01/01/2010

DOI:

10.26481/umamet.2010066

Document Version:

Publisher's PDF, also known as Version of record

\section{Please check the document version of this publication:}

- A submitted manuscript is the version of the article upon submission and before peer-review. There can be important differences between the submitted version and the official published version of record.

People interested in the research are advised to contact the author for the final version of the publication, or visit the DOI to the publisher's website.

- The final author version and the galley proof are versions of the publication after peer review.

- The final published version features the final layout of the paper including the volume, issue and page numbers.

Link to publication

\footnotetext{
General rights rights.

- You may freely distribute the URL identifying the publication in the public portal. please follow below link for the End User Agreement:

www.umlib.nl/taverne-license

Take down policy

If you believe that this document breaches copyright please contact us at:

repository@maastrichtuniversity.nl

providing details and we will investigate your claim.
}

Copyright and moral rights for the publications made accessible in the public portal are retained by the authors and/or other copyright owners and it is a condition of accessing publications that users recognise and abide by the legal requirements associated with these

- Users may download and print one copy of any publication from the public portal for the purpose of private study or research.

- You may not further distribute the material or use it for any profit-making activity or commercial gain

If the publication is distributed under the terms of Article $25 \mathrm{fa}$ of the Dutch Copyright Act, indicated by the "Taverne" license above, 


\section{Maastricht University}

Franz Dietrich, Kai Spiekermann

Epistemic Democracy with Defensible Premises

$\mathrm{RM} / 10 / 066$

\section{METEOR}

Maastricht University School of Business and Economics

Maastricht Research School of Economics

of Technology and Organization

P.O. Box 616

NL - 6200 MD Maastricht

The Netherlands 


\title{
Epistemic Democracy with Defensible Premises
}

\author{
Franz Dietrich \& Kai Spiekermann* \\ London School of Economics \\ October 2010 (minor revisions later)
}

\begin{abstract}
The contemporary theory of epistemic democracy often draws on the Condorcet Jury Theorem to formally justify the 'wisdom of crowds'. But this theorem is inapplicable in its current form, since one of its premises - voter independenceis notoriously violated. This premise carries responsibility for the theorem's misleading conclusion that 'large crowds are infallible'. We prove a more useful jury theorem: under defensible premises, 'large crowds are fallible but better than small groups'. This theorem rehabilitates the importance of deliberation and education, which appear inessential in the classical jury framework.
\end{abstract}

$\mathrm{T}$ he Condorcet Jury Theorem (CJT) looks back on a remarkable career. Discovered by Nicolas de Caritat, Marquis de Condorcet, in 1785, first proved formally by Laplace in 1812 (Ben-Yashar \& Paroush 2007, 190), then long forgotten and finally rediscovered by Duncan Black (Black 1958, Grofman \& Feld 1988), the CJT has now taken centre stage in epistemic conceptions of democracy and in debates in social epistemology. Propelled by claims in the popular literature that crowds can be 'wise' (Surowiecki 2004) and mobs 'smart' (Rheingold 2002), the CJT is now again widely discussed across various disciplines, even beyond academic circles. This career is surprising for a theorem that - in its basic form and applied naively - rests on implausible premises and leads to implausible conclusions, as we will show.

Roughly speaking, the CJT arrives at two conclusions concerning a group decision between two alternatives, where one alternative is objectively correct or better. First, the larger the group gets, the more likely is a correct majority decision. This is the CJT's non-asymptotic conclusion. Second, the probability of a correct majority decision converges to one as the group size tends to infinity. This is the CJT's asymptotic conclusion. Put roughly: larger groups make better decisions, and very large groups are infallible. It is worth reflecting on these results. While we think that the non-asymptotic conclusion is plausible for many setups and can be defended, the asymptotic conclusion is more than dubious. If the asymptotic conclusion applied directly to modern democracies with their large populations, these democracies would

\footnotetext{
*Kai Spiekermann would like to emphasize that the formal results in this paper are the work of Franz Dietrich.
} 
be essentially infallible when making decisions between two alternatives by simple majority.

What went wrong? The CJT rests on two central premises. Stated roughly, the first premise is that the voters vote independently from each other, and the second premise is that each voter is competent, i.e., is more likely to vote for the correct than the incorrect alternative (both premises will later be spelt out more carefully and then revised). Our negative finding is the implausibility of the premises - specifically, the independence premise - which explains the implausible asymptotic conclusion of infallible large groups. The non-asymptotic conclusion that larger groups perform better, by contrast, is plausible, but is currently relying on implausible premises and therefore left hanging without support. Our negative finding can be summed up in a table:

\begin{tabular}{c|c|} 
& Plausible? \\
\hline Condorcet's premises & No \\
\hline The asymptotic conclusion & No \\
\hline The non-asymptotic conclusion & Yes
\end{tabular}

Our positive finding consists in a revision of Condorcet's premises. The new, more plausible premises lead to the new, plausible asymptotic conclusion that large groups are fallible. They also offer a justification for the old non-asymptotic conclusion, which previously rested on implausible premises. This will put us in a new position:

\begin{tabular}{c|c|} 
& Plausible? \\
\hline The new premises & Yes \\
\hline The new asymptotic conclusion & Yes \\
\hline The non-asymptotic conclusion (unchanged) & Yes \\
\hline
\end{tabular}

In fact, the CJT rests on yet another assumption, which is implicit in the classical framework: people vote sincerely. This implicit premise is no less problematic than the explicit premises. It was uncovered in the 1990ies and is being addressed in an ongoing literature. While much progress has already been achieved along this dimension of strategic voting, the problems underlying the explicit premises - specifically, the premise of independent voting - have not yet been understood in a systematic and foundational way. Most believe that 'something' is wrong with Condorcet's premises, but confusion prevails over how to conceptualize and solve the problem. Addressing this is important also because the new literature on strategic voting and the CJT, despite the progress over the classical CJT literature along the strategic voting dimension, still rests on the problematic independence premise (applied now to voters' private information rather than their votes). Since the strategic voting aspect is orthogonal to the problems related to Condorcet's explicit premises, it is not addressed in the present analysis, though a few more remarks on strategic voting will follow below.

This introduction is followed by six sections. Section 1 begins by laying out the classical CJT as it is often cited and applied in the contemporary literature. In section 2 we show that the classical independence assumption cannot be justified and will typically be false, and propose a new, improved independence assumption. The 
revision of the independence assumption requires using a new competence assumption, which is provided in section 3. Section 4 contains our core positive result, a new jury theorem based on the new independence and competence assumptions. We point out that the classical CJT is nothing but a special case of our new theorem. In section 5 we highlight two important implications of the new theorem for the theory of epistemic democracy: deliberation and education may be crucial for the epistemic performance of groups, whereas the classical CJT suggests that they are inessential-since 'crowds are infallible' even without them-or even dangerous - since deliberation threatens voter independence. Section 6 draws conclusions.

\section{Condorcet's Jury Theorem Recapitulated}

We begin by stating and explaining the classical CJT before criticizing its assumptions in the next sections. The first statement can be found in Condorcet (1785), a translation of most parts in Sommerlad and McLean (1989). The theorem has been stated in many variants; an influential one is provided by Grofman et al. (1983). Among the most important applications in political philosophy are Grofman and Feld's (1988) use of the CJT to interpret Rousseau's 'general will' (cf. Estlund et al. 1989) and, of course, the link between the CJT and epistemic conceptions of democracy (Cohen 1986, Gaus 1997, List and Goodin 2001, Estlund 2008). A link between the CJT, judgement aggregation and social epistemology is offered by Bovens and Rabinowicz (2006) and List (2005). Several important generalizations of the classical CJT have been proposed. For instance, Owen et al. (1989) prove that the asymptotic part also holds if judges are heterogeneous in competence, and Atkinson \& Romeijn (forthcoming) analyse cases of unknown competence. Most relevant for our purposes are contributions that discuss the problematic assumption of independence. Quite a few papers recognize that independence is difficult to meet (for instance Nitzan and Paroush 1984, Shapley and Grofman 1984, Boland 1989, Boland et al. 1989, Berg 1993, Estlund 1994, Spiekermann and Goodin 2010, Kaniovski forthcoming). Among the most systematic treatments of independence violations is the work of Krishna Ladha $(1992,1993,1995)$, who shows that shared information typically leads to correlated votes and offers jury theorems for correlated votes. Unlike this literature, we shall develop a causal, network-theoretic account of voter dependence. It is inspired by, but significantly goes beyond Dietrich and List (2004) and Dietrich (2008), who also employ causal network reasoning but only focus on special cases of independence violations.

We now give a precise rendering of the classical CJT. Let there be a group of individuals, labelled $i=1,2,3, \ldots$ The size of the group (electorate) is any number $n$, which we assume to be odd to avoid ties under majority voting. The group has to decide between two alternatives, labelled 0 and 1. Exactly one of these alternatives is 'correct', 'right' or 'better'. ' We will use the attribute 'correct' from now on. The correct alternative is called the state (of the world) and is typically denoted $x$. The

\footnotetext{
${ }^{1}$ Many different notions of objectivity are compatible with that assumption, as long as the right, correct or better answer is a fact that is determined entirely independently from the votes of the individuals. This excludes procedural notions of rightness, where the 'right' solution is right just because it was arrived at by applying the appropriate procedure.
} 
state is generated by a random variable $\mathbf{x}$, taking the values 0 and 1 , each with positive probability. Each person votes for one alternative, abstentions are not allowed. For each voter $i$ we consider an event $R_{i}$, the event that voter $i$ votes correctly. (Some authors take the votes rather than the correct voting events $R_{i}$ as primitives of the model. This makes no difference since the votes and the correct voting events are interdefinable given the state $\mathbf{x} .^{2}$ ) We write $M_{n}$ for the event that a majority of an $n$-member electorate votes correctly. ${ }^{3}$

Classical Independence. Informally, the correct voting events are independent given the state. Formally, $R_{1}, R_{2}, \ldots$ are independent conditional on $\mathbf{x}$.

Classical Competence. Informally, the probability of correct voting given any state exceeds $\frac{1}{2}$ and is the same across voters. Formally, for each state $x \in\{0,1\}, \operatorname{Pr}\left(R_{i} \mid x\right)$ exceeds $\frac{1}{2}$ and does not depend on $i$ (but possibly on $x$ ). ${ }^{4}$

Condorcet Jury Theorem. Suppose Classical Independence and Classical Competence. As the group size increases, the probability $\operatorname{Pr}\left(M_{n}\right)$ that a majority votes correctly (i) increases, and (ii) converges to one.

We now explain the two conditions and the theorem in turn. Events are (conditionally) independent if learning that some of them occurred does not change the (conditional) probability that others occurred. ${ }^{5}$ In our case, the probability that some voters vote correctly (conditional on a state) is not influenced by learning that some other voters vote correctly. An analogy with coin tossing might help. Suppose a coin is tossed independently many times, one toss for each voter. 'Heads' means voting for alternative 1 , 'tails' means voting for 0 . The coin is not just any coin but a predictor coin whose shape is influenced by the state of the world: it is biased towards the correct outcome, in analogy to Classical Competence. The tosses (votes) are not independent unconditionally, since from the outcome of some of the tosses we learn something about the coin shape, and hence about the other tosses. However, in analogy to Classical Independence the tosses are independent conditional on the state, since once we know the state, we know the shape of the coin, so that tosses do not tell us anything new about the coin shape and hence about other tosses.

With the two premises in place, we can turn to the theorem itself. The CJT is nothing but an application of the law of large numbers. In terms of our example, if the predictor coin is thrown very often, it becomes exceedingly likely that the majority of results will be correct, and this probability converges to 1 as the number

\footnotetext{
${ }^{2}$ From $R_{i}$ one can define $i$ 's vote as the random variable $\mathbf{v}_{i}$ in $\{0,1\}$ which matches the state $\mathbf{x}$ in the event $R_{i}$ and differs from $\mathbf{x}$ otherwise. Conversely, if one were to start from $\mathbf{v}_{i}$ one could define $R_{i}$ as the event that $\mathbf{v}_{i}=\mathbf{x}$.

${ }^{3}$ The event $M_{n}$ can be written as $\cup_{I \subseteq\{1, \ldots, n\}: \# I>n / 2} \cap_{i \in I} R_{i}$, because $M_{n}$ means that there is some set of individuals $I \subseteq\{1, \ldots, n\}$ with more than $n / 2$ members such that all individuals $i$ in $I$ vote correctly, i.e., such that $\cap_{i \in I} R_{i}$ obtains.

${ }^{4}$ All probabilistic statements refer to a probability measure $\operatorname{Pr}$ (defined over some underlying algebra of events).

${ }^{5}$ Formally, events are independent if for any (finite) number of them the probability that they occur jointly equals the product of their probabilities. Conditional independence is defined analogously, with probabilities replaced by conditional probabilities.
} 
of tosses tends to infinity. ${ }^{6}$ Large groups are essentially infallible, even if its members are only slightly competent (Goodin and Spiekermann 2010). For instance, with competence only $p=0.51$, a group of 100,000 voters - which is still small for a modern democracy — would be correct in majority with a probability of about 0.99999999987 . This prediction of infallibility for dichotomous factual choices does not withstand empirical scrutiny and will be revised in due course.

Importantly, this framework does not distinguish between someone's vote and his sincere judgment. These two can come apart, since the sincere voting profile may not form a Nash equilibrium (in a suitably defined $n$-player Bayesian game with private information). This fact was long overlooked, presumably because it was taken for granted that strategic voting could not arise when all voters share the same preference for correct collective decisions; it was brought to light in seminal work by AustenSmith and Banks (1996), Feddersen and Pesendorfer (1998), Coughlan (2000), and many others. As much as the literature's current concern for strategic-voting-related shortcomings of the classical model is justified, it might have distracted the attention from the shortcomings of Condorcet's explicit premises, notably Classical Independence. Indeed, as mentioned in the introduction, the strategic-voting literature on the CJT takes independence of private information for granted, while becoming more and more sophisticated on other dimensions. The current paper returns to the origins by analysing and revising Condorcet's two explicit premises. Pursuing two goals at once is never a good idea, and therefore we do not address strategic voting here. But how is one to read our paper in light of the modern insights about strategic voting? Either, one simply assumes that people's votes match their sincere judgments. ${ }^{7}$ Or, if one feels uncomfortable with this assumption, one may reinterpret $R_{i}$ as the event that voter $i$ 's private pre-strategic judgment is correct (whether or not it matches $i$ 's vote), and $M_{n}$ as the event that a majority holds the correct private judgment. Under the latter interpretation, this paper is not about voting but about private opinion formation and the majority opinion; and the insights gained here about private opinion formation would have to be combined with the modern insights about strategic voting in order to form a complete analysis of epistemic aggregation.

\section{Common Causes and the Failure of the Classical In- dependence Assumption}

In this section we show that Classical Independence typically does not hold in realworld decision problems and needs to be revised. Our critique of Classical Independ-

\footnotetext{
${ }^{6}$ Throughout, the term 'increasing' is to be understood as 'weakly increasing'; that is, later values are at least as large as (but not necessarily larger than) earlier values.

${ }^{7}$ Under some specifications of the voters' utilities, this typically implies that at least some voters vote irrationally. These specifications make a voter's utility depend only on the pair of the state and the outcome (majority vote), where utility is high when the outcome matches the state and low otherwise. However, as is often overlooked, voting sincerely is typically rational as soon as voters also care at least slightly about whether their own vote is sincere (for when maximising expected utility the sincerity concern outweighs the outcome-oriented concern since the probability of pivotality is typically very small). Here, the sincere voting assumption of the classical CJT is compatible with game-theoretic rationality.
} 
ence does not take the common line of pointing out that voters can influence each other; rather we show that Classical Independence is questionable even in the absence of any such causal influences between voters. We start by developing an example that we shall use repeatedly in due course. This will be followed by our core argument against Classical Independence: the observation that voters are typically influenced by common causes and therefore not independent in the classical sense. Our New Independence assumption responds to this problem.

Imagine a government relying on a group of economic advisors. Towards the end of 2007, when the US housing market starts dropping, the government wants to know whether a recession is imminent. It asks all advisors and adopts the majority view. To ensure that the experts do not influence each other, safeguards are in place to prevent any communication between them. If the classical CJT applied, we could conclude that the probability of a correct majority vote converges to 1 as more and more advisors are consulted. But this conclusion is unlikely to be true because Classical Independence is typically violated even though the experts do not communicate. To see this, consider a few examples. First, if all economists rely on the same publicly available evidence, then this evidence will usually cause them to vote in the same way. For instance, if all the evidence misleadingly suggests healthy growth (with the evidence indicating, say, that banks have much healthier balance sheets than they actually have) while a bank crash is already around the corner, then most reasonable economists will be wrong in their prediction. The votes are then dependent 'through' consulting the same evidence. More precisely, given for instance that alternative 1 is correct, incorrect votes for 0 by some voters raise the probability of misleading evidence, which in turn raises the probability that other voters also vote incorrectly, a violation of independence. Second, if all economists rely on the same theoretical assumptions for the interpretation of the evidence (such as low correlations between market prices of certain credit default swaps), this common influence is likely to induce dependence between the votes. In the extreme, either all get it right or all get it wrong. Finally, if the experts are more likely to make wrong predictions in weather that gives headaches, then weather creates dependence between votes. Again, in the extreme either all get it right or all get it wrong (and have headaches).

In all these examples, the economic experts are not classically independent and therefore the classical CJT cannot be applied. At the same time, the experts are independent in a different sense. They are independent if we hold all the common causes fixed, or, in different words, if we conditionalize on all the common causes that influence them. Given the economists's particular common evidence, their common background theories, the commonly experienced weather, and all other common causes, their judgements are independent.

We now introduce our new conception of independence more systematically, drawing on the well-established theory of causal networks. The classical independence of votes can be undermined when a common cause influences all voters. This follows from Reichenbach's influential common cause principle, which is usually understood thus: a more frequent than expected coincidence between a set of phenomena, which do not affect each other, is due to (possibly hidden) common causes, and the phenomena become independent once we conditionalize on these common causes (Reichenbach 1956, 159-60). Slightly more precisely: 
Common Cause Principle (CCP). Phenomena which do not causally affect each other are probabilistically independent conditional on their common causes, but usually dependent unconditionally in the presence of common causes. ${ }^{8}$

One can represent common causes graphically, as in figure 1a. Figure 1a is a very simple causal network, depicting the causal relations between a set of variables. A node signifies a phenomenon, mathematically represented as a random variable. An arrow signifies a causal influence in the direction of the arrow. ${ }^{9}$ For simplicity, this and all other figures show only the first two votes, labelled $\mathbf{v}_{1}$ and $\mathbf{v}_{2}$. Both votes are causally influenced by a common cause $\mathbf{c}$ (common causes are shown as grey nodes in all figures). If, for instance, the problem is to judge whether a defendant is guilty, then the common cause $\mathbf{c}$ in figure 1a could be a commonly observed witness report, finger print, or other shared evidence; in all these examples, $\mathbf{c}$ is causally influenced by the fact $\mathbf{x}$ of whether the defendant is guilty, as represented by the arrow pointing from $\mathbf{x}$ towards $\mathbf{c} .{ }^{10}$ The effect of $\mathbf{c}$ on votes is indicated by the arrows from $\mathbf{c}$ towards each vote. Note that there are no arrows between any votes, indicating that the votes do not have a causal influence on each other. But even though the votes are causally independent, they are not probabilistically independent due to the common cause $\mathbf{c}$.

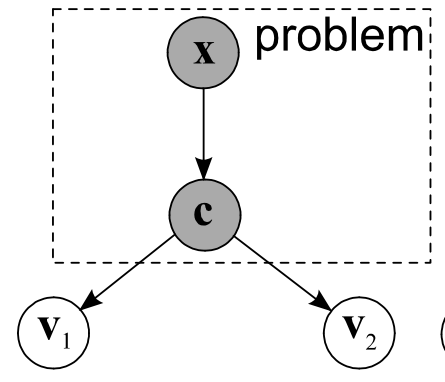

(a)

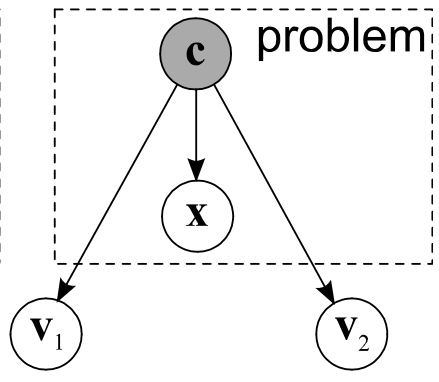

(b)

Figure 1: Two simple causal networks with common causes.

Compare figure $1 \mathrm{a}$ with $1 \mathrm{~b}$. In $1 \mathrm{~b}$, the two votes $\mathbf{v}_{1}$ and $\mathbf{v}_{2}$ are still influenced by the common cause, but now the direction of causality between common cause and state is swapped so that $\mathbf{c}$ is affecting $\mathbf{x}$. To make this plausible, consider an example. Suppose the task for the voters is to decide whether the streets are wet in Windsor, but all voters are placed in central London. Further suppose that the voters follow a simple heuristic: they predict that the streets are wet in Windsor if and only if the weather is bad in London. In that case, c could be seen as the weather pattern over

\footnotetext{
${ }^{8}$ Usually, the common cause principle is stated for only two phenomena. We state it here for an arbitrary number of phenomena such as many votes. The CCP is developed more formally in the theory of Bayesian networks (e.g., Spirtes, Glymour and Scheines 1993, and Pearl 2000.

${ }^{9}$ We are not going to discuss causal and Bayesian networks in detail here. For a thorough introduction see Pearl 2000, ch. 1. Formally, a causal network is a so-called directed acyclic graph, which consists of a set of nodes (carrying random variables) and a set of directed arrows between distinct nodes (representing causal relevance between variables) such that there is no directed cycle of arrows.

${ }^{10}$ While votes are dichotomous random variables, taking only the values 0 or 1 , a cause such as $\mathbf{c}$ need not be dichotomous. For instance, shared evidence can take more than two forms.
} 
southeast England, influencing both the votes and the state. So, in this network the common cause $\mathbf{c}$ (the weather) influences the state $\mathbf{x}$ (dry or wet streets in Windsor), and not the other way round, unlike in figure $1 \mathrm{a}$.

Let us consider a more complicated example. In figure 2 we include a number of

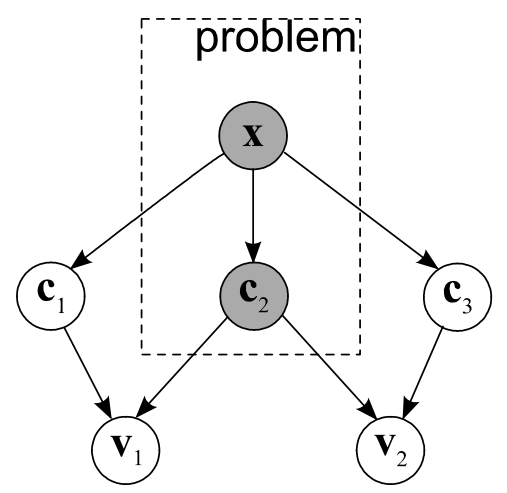

Figure 2: A causal network with private and common causes.

causes from $\mathbf{c}_{1}$ to $\mathbf{c}_{3}$. We can now distinguish between private and common causes. In this causal network, the cause $\mathbf{c}_{2}$ affects both votes and hence is a common cause. $\mathbf{c}_{1}$ and $\mathbf{c}_{3}$, by contrast, are private causes because they only affect one vote each. By definition, common causes are nodes that have directed paths of arrows to more than one vote node. This is why $\mathbf{x}$ is also a common cause (and is therefore displayed in grey): it has paths running to $\mathbf{v}_{1}$ (through $\mathbf{c}_{1}$ or $\mathbf{c}_{2}$ ) and to $\mathbf{v}_{2}$ (through $\mathbf{c}_{2}$ or $\mathbf{c}_{3}$ ).

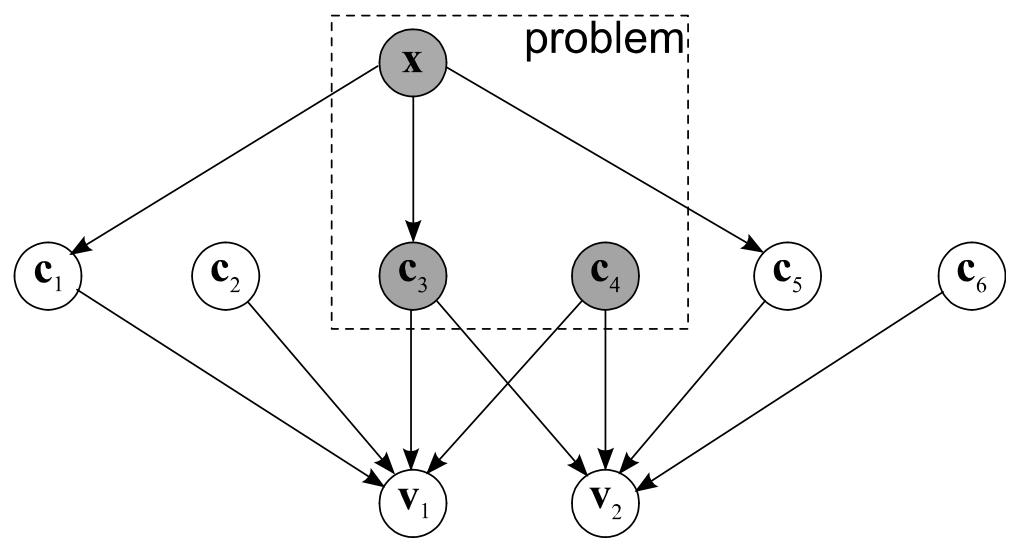

Figure 3: A causal network with private and common causes, some of which are non-evidential.

Figure 3 shows another, slightly more complicated, causal network. As before, we have common causes $\left(\mathbf{c}_{3}, \mathbf{c}_{4}\right.$ and $\left.\mathbf{x}\right)$ and private causes $\left(\mathbf{c}_{1}, \mathbf{c}_{2}, \mathbf{c}_{5}\right.$ and $\left.\mathbf{c}_{6}\right)$. But in contrast to the previous figures, we can now distinguish between non-evidential causes that are not related to the state $\left(\mathbf{c}_{2}, \mathbf{c}_{4}\right.$ and $\left.\mathbf{c}_{6}\right)$ and evidential causes that are. Nonevidential causes are factors that influence voters causally but do not provide them 
with evidence as to the correct alternative. These could be common non-evidential causes (such as the weather influencing all our economic advisors in the example above) or private non-evidential causes (such as a domestic argument influencing the judgment of one advisor).

In all figures shown so far the votes $\mathbf{v}_{1}$ and $\mathbf{v}_{2}$ are not state-conditionally independent because of common causes (other than $\mathbf{x}$ ). That votes have such common causes is not in any way unusual. To assume that there are none, as the classical CJT does, is to assume a highly construed, artificial decision problem that is unlikely to occur in real-life settings. Thus, Classical Independence is typically violated. This means that the conclusions of the CJT rest on a (typically) false premise. The upshot is that the independence assumption must be revised.

We now introduce a more defensible independence assumption. The CCP implies that events are independent conditional on all their common causes. So independence of votes can be achieved if we conditionalize in the right way. The classical CJT conditionalized on $\mathbf{x}$ only. But to catch all the common causes, both evidential and non-evidential, we propose to conditionalize on the decision problem at hand. The decision problem is a description of all relevant features of the task the group faces. The problem captures not only the state of the world but also all relevant circumstances, which we interpret as all common causes of the votes, thus excluding purely individual factors. We therefore conditionalize on the state of the world and all common causes, as indicated by the dashed rectangles in figures 1-3. Both components of a decision problem are indispensable. Defining the problem without the circumstances does not work, as we have seen. Defining it without the state $\mathbf{x}$ is not plausible either, since this would leave the correct answer indeterminate. Often, $\mathbf{x}$ is itself a common cause anyhow, as in figures 1a, 2 and 3 but not in figure 1b.

We now return to the formal model, leaving the motivational discussion based on causal networks behind. As far as model ingredients are concerned, only one amendment of the classical model is needed. We keep the correct voting events $R_{1}, R_{2}, \ldots$ but dispose of the state variable $\mathbf{x}$ in favour of a new random variable $\pi$, the problem, whose realisations are the various possible problems $\pi$ that the group might face. ${ }^{11}$ Interpretationally, the problem captures not just the state of the world but also the circumstances (common causes) the voters face.

We are now ready to state our revised independence premise.

New Independence. The events $R_{1}, R_{2}, \ldots$ that voters $1,2, \ldots$ vote correctly are independent conditional on the problem $\pi{ }^{12}$

This assumption is far more defensible than the classical one; by conditionalizing on the problem, we fix all those circumstances which, if left variable, could lead to voter correlation. In figure 3 , for instance, by conditionalizing on the problem one conditionalizes on all the common causes, both evidential (like $\mathbf{c}_{3}$ in figure 3 ) and

\footnotetext{
${ }^{11}$ Problems can (and will in real life) be highly complex objects. Our model accounts for such complexity without loss of parsimony.

${ }^{12}$ Independence conditional on the random variable $\boldsymbol{\pi}$ means that for any value which $\boldsymbol{\pi}$ may take (with positive probability) there is independence conditional on $\boldsymbol{\pi}$ taking this value. This definition assumes that $\boldsymbol{\pi}$ is discrete, i.e., takes only countably many values. For the general definition of independence conditional on an arbitrary random variable we refer the reader to standard textbooks.
} 
non-evidential (like $\mathbf{c}_{4}$ in figure 3 ), so that the two events become probabilistically independent. In the terminology of causal networks, the common causes 'screen off' the votes from each other, so that conditionalizing on these causes removes any correlations between votes.

For the interested reader, appendix A briefly sketches the more formal networktheoretic foundation of New Independence; there, the network-theoretic motivation given above is turned into a theorem.

\section{The Need to Revise the Competence Assumption}

The Classical Competence assumption is not unreasonable; in fact it is plausibly true. It tells us that the voters are more likely to vote correctly than incorrectly. But when combined with our New Independence assumption (rather than with the unrealistic classical one) it doesn't imply that large groups are better than small ones in their majority judgments.

Consider our group of economic advisors again. It is plausible to assume that each economist is on average better than random in answering economic yes/no questions such as whether a recession is imminent. However, we also know that economists are often not competent when considering one single problem, because some problems are more difficult than others. For instance, very few economists correctly predicted that the initially quite limited banking crisis of 2008 would trigger a major recession in 2009. With hindsight we have learned that predicting this crisis was a difficult problem because economists faced misleading data and worked with questionable or incorrect assumptions. In many other settings, predicting a recession is easy (or at least easier) and economists are more likely to be correct in their predictions. Economists can be competent on average, as demanded by the Classical Competence assumption, without being competent on a difficult problem.

Classical Competence may hold, but since our New Independence assumption conditionalizes on the problem, we will need a problem-specific notion of competence. To show this need we give a stylized example in which large groups are much worse than small ones, despite classical voter competence. This demonstrates that the conclusions of the classical CJT may fail if Classical Independence is replaced with New Independence while retaining Classical Competence. Suppose there are only two types of problems, 'easy' and 'difficult' ones, where each type is equally likely to occur. Each voter $i$ is correct on any easy problem with probability 0.99 , and on any difficult one with probability 0.49 . That is,

$$
\operatorname{Pr}\left(R_{i} \mid \pi\right)= \begin{cases}0.99 & \text { for every easy problem } \pi \\ 0.49 & \text { for every difficult problem } \pi\end{cases}
$$

First note that each voter $i$ is competent in the sense that he votes correctly with

probability $\operatorname{Pr}\left(R_{i}\right)=\frac{1}{2} \times 0.99+\frac{1}{2} \times 0.49=0.74$. Also the two state-conditional competence parameters, $\operatorname{Pr}\left(R_{i} \mid \mathbf{x}=1\right)$ and $\operatorname{Pr}\left(R_{i} \mid \mathbf{x}=0\right)$, exceed $\frac{1}{2}$ under mild additional conditions (essentially, there shouldn't be a too high correlation between problem type and state). So, Classical Competence holds. Despite the voters' high competence, the majority's competence in a large group is low and well below individual 
competence. Indeed, the probability that the majority is correct is

$$
\operatorname{Pr}\left(M_{n}\right)=\frac{1}{2} \times \operatorname{Pr}\left(M_{n} \mid \boldsymbol{\pi} \text { is easy }\right)+\frac{1}{2} \times \operatorname{Pr}\left(M_{n} \mid \boldsymbol{\pi} \text { is difficult }\right),
$$

where the term $\operatorname{Pr}\left(M_{n} \mid \boldsymbol{\pi}\right.$ is easy) is roughly one, but the term $\operatorname{Pr}\left(M_{n} \mid \boldsymbol{\pi}\right.$ is difficult) is roughly $\frac{1}{2}$ if $n$ is small (because voters are only slightly worse than fair coins) but tends to zero as $n$ tends to infinite; ${ }^{13}$ so,

$$
\operatorname{Pr}\left(M_{n}\right) \approx \begin{cases}\frac{1}{2} \times 1+\frac{1}{2} \times \frac{1}{2}=\frac{3}{4} & \text { for small } n \\ \frac{1}{2} \times 1+\frac{1}{2} \times 0=\frac{1}{2} & \text { for large } n\end{cases}
$$

Large groups are worse here than small groups or single individuals! In terms of our example, asking just one economist would be better than asking many because the majority of the many is increasingly likely to get the difficult problem wrong.

We thus need a new notion of competence: one that is relative to the decision problem. Let us define a voter $i$ 's (problem-specific) competence as $p_{i}^{\boldsymbol{\pi}}=\operatorname{Pr}\left(R_{i} \mid \boldsymbol{\pi}\right)$, the probability that $i$ votes correctly conditional on the problem. Its value depends on the problem; intuitively, it is high for 'easy' problems and low for difficult ones. In the last example, problem-specific competence is 0.99 or 0.49 , depending on whether the problem is easy or difficult. The value taken for a particular problem $\pi$-a particular realisation of $\boldsymbol{\pi}$-is called the competence on $\pi$, denoted $p_{i}^{\pi}=\operatorname{Pr}\left(R_{i} \mid \pi\right)$. Figure 4 gives an example of how the distribution of a voter $i$ 's problem-specific

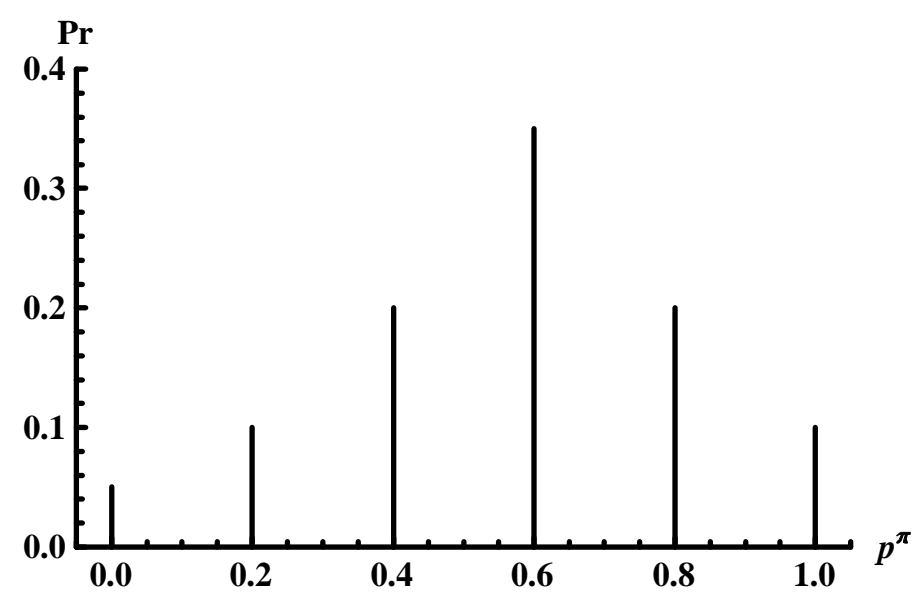

Figure 4: A discrete distribution of problem-specific competence.

competence could look like. There is a $10 \%$ probability of facing a problem on which competence is as high as 1 (i.e., the voter is always right), a $20 \%$ probability of facing a problem on which competence is 0.8 , and so on for the competence levels of 0.6, 0.4, 0.2 and 0. While in figure 4 problem-specific competence follows a discrete distribution (with six possible values), figure 5 shows an example in which problem-

\footnotetext{
${ }^{13}$ It tends to zero because, given a difficult problem, the proportion of correct votes tends to 0.49 by the law of large numbers.
} 


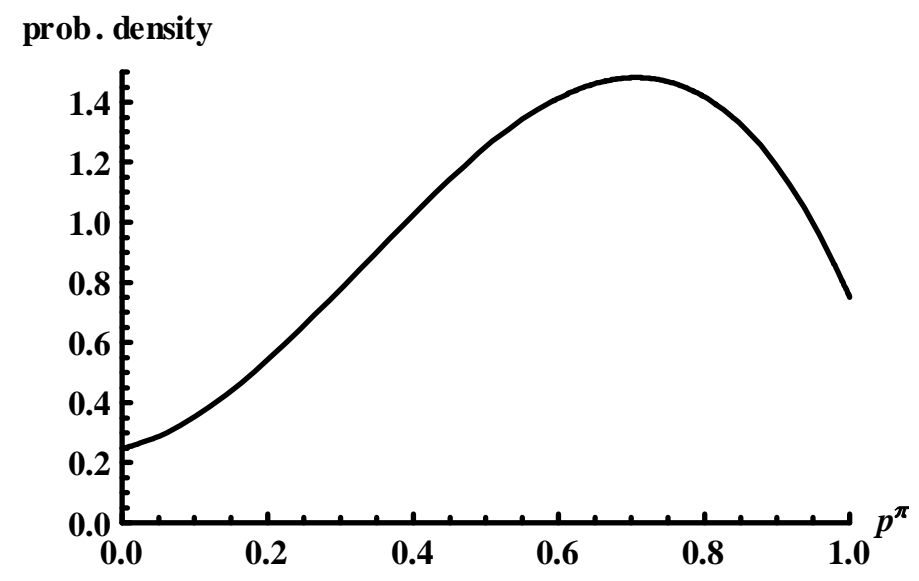

Figure 5: A continuous distribution of problem-specific competence.

specific competence follows a continuous distribution given by a density function over the interval $[0,1]$. Of course, many other discrete or continuous distributions of problem-specific competence are imaginable.

Notice that in figures 4 and 5 the distribution of a voter's problem-specific competence on the interval $[0,1]$ is 'skewed' to the right, so that, informally, he is more likely to face an 'easy' problem (on which competence is high) than a 'difficult' problem (on which competence is low). In short, the voter finds more problems easy than difficult. Clearly, this is a notion of voter 'competence', but a somewhat different one than that required by Classical Competence. This new sense of voter competence is what our New Competence assumption formally requires:

New Competence. Problem-specific competence $p_{i}^{\pi}$ is more likely to be high than low-that is, is $\frac{1}{2}+\epsilon$ with at least as much probability as it is $\frac{1}{2}-\epsilon$, for all $\epsilon>0$ - and is the same for all voters $i$ - that is, $p_{i}^{\pi} \equiv p^{\pi}$. ${ }^{14}$

To paraphrase this condition once more, the problem is more likely to be of the sort on which voters are competent than of the sort on which they are incompetent (where voters are homogeneous in competence, as in the classical setup). Problem-specific competence thus follows a right-skewed distribution on the unit interval $[0,1]$ in the sense that competence levels above $\frac{1}{2}$ are at least as likely than their symmetrically opposed levels below $\frac{1}{2}$. This clearly holds for figures 4 and $5,{ }^{15}$ and of course for many other plausible (discrete or continuous) distributions on the interval $[0,1]$.

\footnotetext{
${ }^{14}$ The clause 'that is, is $\frac{1}{2}+\epsilon \ldots$ for all $\epsilon>0$ ' is stated for the case that problem-specific competence has a discrete distribution (as in figure 4 but not as in figure 5). The general statement, valid for any sort of distribution, reads as follows: 'that is, belongs to $\left[\frac{1}{2}+\epsilon, \frac{1}{2}+\epsilon^{\prime}\right]$ with at least as much probability as it belongs to $\left[\frac{1}{2}-\epsilon^{\prime}, \frac{1}{2}-\epsilon\right]$, for all $\epsilon^{\prime} \geq \epsilon>0$ '. This general condition reduces to the condition in the main text in the discrete case. It reduces to another simple condition in the continuous case with a continuous density function, namely the condition that this density be at least as high at $\frac{1}{2}+\epsilon$ as at $\frac{1}{2}-\epsilon$ for all $\epsilon>0$.

${ }^{15}$ Regarding figure 4 , check that $\operatorname{Pr}\left(p^{\boldsymbol{\pi}}=1\right)=0.1 \geq 0.05=\operatorname{Pr}\left(p^{\boldsymbol{\pi}}=0\right), \operatorname{Pr}\left(p^{\boldsymbol{\pi}}=0.8\right)=0.2 \geq$ $0.1=\operatorname{Pr}\left(p^{\pi}=0.2\right), \operatorname{Pr}\left(p^{\pi}=0.6\right)=0.35 \geq 0.2=\operatorname{Pr}\left(p^{\pi}=0.4\right)$, and $\operatorname{Pr}\left(p^{\pi}=\frac{1}{2}+\epsilon\right)=0 \geq 0=$ $\operatorname{Pr}\left(p^{\pi}=\frac{1}{2}-\epsilon\right)$ for all $\epsilon>0$ such that $\epsilon \neq 0.1,0.3,0.5$. Regarding figure 5 , check that the condition
} 
The New Competence assumption formalizes an idea stated informally at the beginning of the section in terms of our economist example. The economists face easy and difficult problems. Some problems are very hard, for example predicting the global recession of 2009, but fortunately not all problems are like that. If the economists are good economists they find most problems easy and fewer problems difficult. Unlike the Classical Competence assumption, our new assumption makes no explicit statement about the economists' average competence across all problems, or across all problems for which a given state $x$ in $\{0,1\}$ obtains. Instead, we assume that the economists more often have high than low competence.

As expected, our competence assumption fails for the paradoxical scenario discussed earlier in this section, since there a voter's problem-specific competence is less likely to be 0.51 than 0.49 , since $\operatorname{Pr}\left(p^{\pi}=0.51\right)=0$ and $\operatorname{Pr}\left(p^{\pi}=0.49\right)=\frac{1}{2} \cdot{ }^{16}$ The violation of New Competence is the deeper reason for our counterintuitive finding that large groups are worse than single individuals.

\section{The New Jury Theorem}

The New Jury Theorem is based on the New Independence and Competence assumptions. To introduce it, consider again our panel of economic advisors having to predict whether there will be a recession. We assume (from New Independence) that given any problem the events of correct predictions are independent across voters. We also assume (from New Competence) that the prediction problem is more likely to be easy than difficult. The New Jury Theorem states that increasing the group size will increase the probability of a correct majority, sampled across prediction problems. This is the old non-asymptotic conclusion of the CJT, but based on new premises. The New Jury Theorem also states that for very large groups the collective competence no longer approaches one (revising the old, unrealistic asymptotic conclusion). Rather, the asymptotic value now depends on the proportion of easy problems.

More precisely, the new theorem goes as follows:

New Jury Theorem. Suppose New Independence and New Competence. As the group size increases, the probability that a majority votes correctly (i) increases, and (ii) converges to a value which is less than one if $\operatorname{Pr}\left(p^{\pi}>\frac{1}{2}\right) \neq 1$ (and one if $\left.\operatorname{Pr}\left(p^{\boldsymbol{\pi}}>\frac{1}{2}\right)=1\right)$.

Remark: As the proof shows, the value to which the probability of a correct majority converges is

$$
\operatorname{Pr}\left(p^{\pi}>\frac{1}{2}\right)+\frac{1}{2} \operatorname{Pr}\left(p^{\pi}=\frac{1}{2}\right)
$$

of footnote 14 is satisfied, since the plotted density is at least as high at $\frac{1}{2}+\epsilon$ as at $\frac{1}{2}-\epsilon$ for all $\epsilon>0$.

${ }^{16}$ Note that the support of the competence distribution-namely, the set $\{.49, .99\}$ - seems artificial in that it is very small and not symmetric around the middle $\frac{1}{2}$. In modelling practice, most distributions on $[0,1]$ are symmetric, as they are either continuous and supported by the full interval $[0,1]$ (as in figure 5) or discrete and supported by a regular grid of the form $\left\{\frac{k}{m}: k=0, \ldots, m\right\}$ for a positive integer $m$ (as in figure 4 , where $m=5$ ). As long as the competence distribution has symmetric support, this distribution is plausibly compatible with our New Competence assumption. By definition, the support of a distribution on $[0,1]$ is the minimal topologically closed set $S \subseteq[0,1]$ of probability one, and $S$ is symmetric (around $\frac{1}{2}$ ) just in case $\frac{1}{2}+\epsilon \in S \Leftrightarrow \frac{1}{2}-\epsilon \in S$ for all $\epsilon>0$. 
the probability that the problem is easy plus half of the probability that the problem is on the boundary between easy and difficult.

While proving the non-asymptotic conclusion is not straightforward (see appendix $\mathrm{B}$ ), it is easy to develop an intuition of what is driving the asymptotic conclusion and the remark. We know that voters (our economic advisors, for instance) have a probability greater than $\frac{1}{2}$ to vote correctly on easy problems, smaller than $\frac{1}{2}$ on difficult problems, and equal to $\frac{1}{2}$ on boundary problems. So, by the law of large numbers, in the limit the majority will be correct on easy problems, wrong on difficult problems, and correct with probability $\frac{1}{2}$ on boundary problems. Hence, the limiting probability of the majority being correct, averaged over all problems, is as specified in the remark. For example, if our economic advisors face easy problems $80 \%$, difficult problems $20 \%$, and boundary problems $0 \%$ of the time, then increasing the group of advisors will let their collective competence converge to 0.8 .

The mathematical power and generality of the theorem is that it holds regardless of how we specify the problem variable $\boldsymbol{\pi}$. Indeed, although we have suggested a specific interpretation of $\boldsymbol{\pi}$-it captures common causes - we are mathematically free in how we specify it. Let us illustrate this flexibility by considering two very simple specifications, which depart from our suggested interpretation by not capturing the common causes. These 'naive' specifications allow us to recover (in fact, strengthen) the classical CJT in two variants. For under these specifications our premises and conclusions reduce to the classical ones.

First, suppose $\boldsymbol{\pi}$ takes only two values, 0 and 1, representing the state of the world. So $\boldsymbol{\pi}$ plays precisely the same role as the state in the classical CJT; we accordingly write $\mathbf{x}$ for $\boldsymbol{\pi}$. To show that we obtain the Classical CJT, note that Classical Independence implies (in fact, is directly equivalent to) New Independence, and Classical Competence implies New Competence, so that our theorem tells us that the probability of a correct majority vote is increasing in group size and (by $\operatorname{Pr}\left(p^{\mathbf{x}}>\frac{1}{2}\right)=1$ ) converges to one, just as in the Classical CJT. In fact, this strengthens the Classical CJT since New Competence is a weaker premise than Classical Competence. ${ }^{17}$

Second, assume even more simply that $\boldsymbol{\pi}$ takes only one value - there is just a single problem. Conditionalizing on this fixed problem is as much as not conditionalizing at all. Therefore, our two premises take a particularly simple form:

- the events $R_{1}, R_{2}, \ldots$ are (unconditionally) independent, and

- competence - the unconditional probability $\operatorname{Pr}\left(R_{i}\right)$ - is at least $\frac{1}{2}$ and the same across voters.

As for our theorem's conclusions, they are here the classical ones: majority competence is increasing in group size and converges to one (unless competence is exactly $\left.\frac{1}{2}\right)$.

\footnotetext{
${ }^{17}$ Because under New Competence a voter's problem-specific ('state-specific') competence $p^{\mathbf{x}}$ need not always exceed $\frac{1}{2}$ as long as it is more likely to be high than low in an appropriate sense. For instance, it could be that $p^{1}=0.6$ and $p^{0}=0.4$, where $\mathbf{x}$ is more likely 1 than 0 .
} 


\section{$5 \quad$ Epistemic Democracy: Deliberation and Education}

Our findings have implications for theories of epistemic democracy. This section develops two issues. First, our framework brings to light the benefits of deliberation by removing the worry that independence could be undermined once our new independence notion is adopted. Second, the framework rehabilitates the importance of individual competence for group performance, and hence of education and other competence-boosting policy measures.

Normatively attractive conceptions of democracy involve interactions of voters before the vote. Indeed, many democratic theorists emphasize the importance of deliberation for democratic legitimacy or the quality of democratic outputs. The problem is that such interactions may undermine independence when construed classically, as many have stressed. Nonetheless, confusion prevails over how deliberation creates dependence, and what this implies for jury theorems. Adrian Vermeule describes the challenge well:

"What is unclear is whether, and to what extent, independence is compromised by deliberation, discussion, or even common social background or professional training. [...] Absent any general account of this, the basic reach of the Jury Theorem is not well understood and no amount of possibility theorems or anecdotes about wise crowds will tell us whether the Theorem is an important tool of political and legal theory or a minor curiosity." (Vermeule 2009, 6-7, reference and footnote omitted)

Our analysis responds to this challenge by offering the required 'general account'in terms of causal networks - and by revising the independence notion so as to conditionalize on all common causes, including deliberation. A somewhat different response would be to stick to Classical Independence and try to enforce it by preventing all deliberation, as discussed by Grofman and Feld (1988) in their seminal work connecting the Condorcet Jury Theorem with Rousseau's 'general will'. Many find this approach normatively unacceptable. Aside from the normative worry, preventing deliberation is only sensible if it is true that deliberation creates dependence. But is it? Jeremy Waldron is guardedly optimistic that it is not:

"The sort of interaction between voters that would compromise independence would be interaction in which voter $\mathrm{X}$ decided in favour of a given option just because voter Y did. [...] But X's being persuaded by $\mathrm{Y}$ in argument or holding itself open to such persuasion does not in itself involve X's deciding to vote one way rather than another because of the way $\mathrm{Y}$ is voting." (Waldron in Estlund et al. 1989, 1327)

Waldron here identifies the paradigmatic case of dependence: one voter following another blindly. Spelled out in our causal-network-theoretic terms, Waldron's point is that persuasion and deliberation by themselves do not undermine independence because they do not constitute causal effects (arrows) between votes but causal effects (arrows) from earlier phenomena c - such as speech acts - to votes. Thus restated, it becomes clear that Waldron's argument is in fact a defense of New rather than 
Classical Independence, since persuasive speech acts will, like other common causes of votes, threaten Classical but not New Independence. Indeed, persuasion counts among the more subtle threats to Classical Independence, working through common causation rather than inter-causation of votes, as illustrated in many of our figures above. Persuasion and deliberation could, for instance, mean that the voters align their theories or the set of evidence they use, thereby introducing powerful common causes that undermine Classical Independence.

David Estlund points out that Classical Independence can sometimes be met even when there are common causes or inter-causation of votes (Estlund 2008, 225). He refers to causal setups where the different effects cancel each other out such that probabilistic independence obtains. It needs to be said, however, that while such settings are logically possible, they are exceedingly rare, especially when many voters are involved. Typically, common causes are ubiquitous, and there is little hope that Classical Independence is preserved after deliberation.

However, deliberation does not necessarily increase voter dependence in the classical sense. It could decrease dependence by reducing the influence of certain other common causes (like room temperature) or direct causal influences between voters. It is therefore not always obvious whether deliberation overall increases or decreases dependence, another reason why the classical CJT literature struggles so much with deliberation. Our framework, by contrast, avoids this fruitless struggle. We give the deliberative process its proper place by including it in the description of the problem $\boldsymbol{\pi}$, and after having conditionalized on this richly described problem, deliberation does not threaten independence anymore.

The classical framework leaves one with the unsatisfactory diagnosis that successful deliberation typically increases voter competence on the one hand, but typically reduces voter independence on the other. Given this unresolved trade-off, the overall effect on group performance could be positive or negative. The diverging claims in the literature about whether deliberation is beneficial demonstrate this all too well. With our New Theorem, by contrast, we can focus exclusively on how deliberation affects (New) Competence. In the language of our framework, deliberation is epistemically beneficial if it increases the voters' problem-specific competence (that is, shifts its distribution to the right). If it does, it also raises the probability that a majority is correct. Thus, deliberation should be interpreted as a process that affects the probability distribution of problem-specific competence, without undermining New Independence. For illustration, consider our economic advisors one last time. If they deliberate and exchange evidence or views, they do not thereby threaten New Independence (because this deliberation process is part of the problem we conditionalize on), but they might raise their problem-specific competence, turning difficult into easier problems. Consequently, a group of deliberating economists may perform better because they are more likely to face decisions they tend to get right, while isolated economists may not.

Our framework has another advantage over the classical one. In its policy recommendation, the classical framework puts all the emphasis on increasing the size of the electorate, suggesting that this suffices for optimal group performance. Classically, large groups can be made infallible without increasing individual competence, just by increasing group size. This loses sight of another important aspect of insti- 
tutional design: the improvement of individual competence. Our framework restores the picture, showing that individual competence levels matter considerably, since they determine the upper bound on group performance. Policy measures such as improving education may raise the upper bound. Put bluntly, not just the size but also the quality of crowds matters.

\section{Conclusion}

Condorcet's classical jury theorem has an enormous influence, but its independence assumption is implausible and is responsible for the overly optimistic asymptotic conclusion that 'large groups are infallible'. The non-asymptotic conclusion that 'larger groups perform better', by contrast, is often plausible, but is in need of a new justification, grounded on more defensible premises. We have provided such a justification. Our revised independence assumption does not require independence across all decision problems but independence given the specific problem. This allows us to conditionalize on common causes which would otherwise have induced dependence. This new independence assumption requires a new competence assumption: rather than assuming voter competence on average over all problems, we assume that the voters' problem-specific competence is more often high than low. Based on our two revised premises, our New Jury Theorem retains the classical conclusion that 'larger crowds are wiser' but obtains the new asymptotic conclusion that 'large crowds are fallible'. Specifically, the probability of a correct majority vote converges to the probability that the problem is easy in a technical sense. These conclusions vindicate majoritarian democracy - it is worth listening to many rather than few - without being absurdly optimistic about the correctness of democratic decisions. The move from the classical to our new jury theorem can be summarized in a table:

\begin{tabular}{c|c|c|} 
& Classical Jury Theorem & New Jury Theorem \\
\hline Independence premise & implausible & plausible \\
\hline Competence premise & plausible & plausible \\
\hline Do larger groups perform better? & yes & yes \\
\hline Are very large groups infallible? & yes & no \\
\hline
\end{tabular}

Our theorem leads to different policy implications than the classical one with regard to the importance of deliberation and education. The worry that deliberation threatens voter independence disappears by moving to our new notion of independence, so that one can focus on the potentially beneficial effect of deliberation on voter competence. The importance of promoting voter competence (through education, deliberation and other measures) is rehabilitated. Indeed, while the classical model implies that the level of voter competence is essentially irrelevant - since large enough groups are infallible even if their members are just a little competent - our model implies that the performance of large groups strongly depends on voter competence, as measured by the proportion of decision problems voters find easy (in our technical sense). Overall, we believe our findings give more credibility to epistemic arguments for democracy based on jury theorems. 


\section{References}

Atkinson, D. \& Romeijn, J. (forthcoming), 'A Condorcet jury theorem for unknown juror competence', Politics, Philosophy, and Economics.

Austen-Smith, D. \& Banks, J. (1996), 'Information Aggregation, Rationality, and the Condorcet Jury Theorem', American Political Science Review, 90, 34-45.

Ben-Yashar, R. \& Paroush, J. (2000), 'A nonasymptotic Condorcet jury theorem', Social Choice and Welfare 17(2), 189-199.

Berg, S. (1993), 'Condorcet's jury theorem: dependency among voters', Social Choice and Welfare 10, 87-95.

Black, D. (1958), The Theory of Committees and Elections, Cambridge University Press, Cambridge.

Boland, P. J. (1989), 'Majority systems and the Condorcet jury theorem', Journal of the Royal Statistical Society. Series D (The Statistician) 38(3), 181-189.

Boland, P. J.; Proschan, F. \& Tong, Y. (1989), 'Modelling dependence in simple and indirect majority systems', Journal of Applied Probability 26(1), 81-88.

Bovens, L. \& Rabinowicz, W. (2006), 'Democratic Answers to Complex Questions an Epistemic Perspective', Synthese 150, 131-153.

Cohen, J. (1986), 'An Epistemic Conception of Democracy', Ethics 97(1), 26-38.

Condorcet, Marquis, D. (1785), Essai sur l'application de l'analyse á la probabilité des décisions rendues á la pluralité des voix.

Coughlan, P. J. (2000), 'In defense of unanimous jury verdicts: mistrials, communication and strategic voting', American Political Science Review, 94, 375-94.

Dietrich, F. (2008), 'The premises of Condorcet's jury theorem are not simultaneously justified', Episteme 58(1), 56-73.

Dietrich, F. \& List, C. (2004), 'A model of jury decisions where all jurors have the same evidence', Synthese 142, 175-202.

Estlund, D.; Waldron, J.; Grofman, B. \& Feld, S. L. (1989), 'Democratic Theory and the Public Interest: Condorcet and Rousseau Revisited', American Political Science Review 83(4), 1317-1340.

Estlund, D. M. (2008), Democratic Authority: A Philosophical Framework, Princeton University Press, Princeton.

Estlund, D. M. (1994), 'Opinion leaders, independence, and Condorcet's Jury Theorem', Theory and Decision 36(2), 131-162.

Feddersen, T. \& Pesendorfer, W. (1998), 'Convicting the innocent: the inferiority of unanimous jury verdicts under strategic voting', American Political Science Review, 92, 23-36.

Gaus, G. (1997), 'Does democracy reveal the voice of the people? Four takes on Rousseau', Australasian Journal of Philosophy 75(2), 141-162.

Goodin, R. E. \& Spiekermann, K. (2010), 'Epistemic Aspects of Representative Government', unpublished manuscript.

Grofman, B. \& Feld, S. L. (1988), 'Rousseau's General Will: A Condorcetian Perspective', American Political Science Review 82(2), 567-576.

Grofman, B.; Owen, G. \& Feld, S. L. (1983), 'Thirteen Theorems in Search of the Truth', Theory and Decision 15, 261-278.

Kaniovski, S. (2010), 'Aggregation of correlated votes and Condorcet's Jury The- 
orem', Theory and Decision 69(3), 453-468.

Ladha, K. K. (1995), 'Information pooling through majority-rule voting: Condorcet's jury theorem with correlated votes', Journal of Economic Behavior ES Organization 26(3), 353-372.

Ladha, K. K. (1993), 'Condorcet's jury theorem in light of de Finetti's theorem', Social Choice and Welfare 10(1), 69-85.

Ladha, K. K. (1992), 'The Condorcet Jury Theorem, Free Speech, and Correlated Votes', American Journal of Political Science 36(3), 617-634.

List, C. (2005), 'The Probability of Inconsistencies in Complex Collective Decisions', Social Choice and Welfare 24(1), 3-32.

List, C. \& Goodin, R. E. (2001), 'Epistemic Democracy: Generalizing the Condorcet Jury Theorem', Journal of Political Philosophy 9(3), 277-306.

Nitzan, S. \& Paroush, J. (1984), 'The significance of independent decisions in uncertain dichotomous choice situations', Theory and Decision 17(1), 47-60.

Owen, G.; Grofman, B. \& Feld, S. L. (1989), 'Proving a distribution-free generalization of the Condorcet Jury Theorem', Mathematical Social Sciences 17(1), $1-16$.

Pearl, J. (2000), Causality: models, reasoning and inference, Cambridge University Press, Cambridge.

Reichenbach, H. (1956), The direction of time, University of California Press, Berkely. Rheingold, H. (2002), Smart mobs: the next social revolution, Perseus Publishing, Cambridge, MA.

Shapley, L. \& Grofman, B. (1984), 'Optimizing group judgmental accuracy in the presence of interdependencies', Public Choice 43(3), 329-343.

Sommerlad, F. \& McLean, I. (1989), 'The Political Theory of Condorcet', Social Studies Faculty Centre Working Paper, Oxford University, 1/89.

Spiekermann, K. R Goodin, R. E. (2010), 'Courts of Many Minds', APSA 2010 Annual Meeting Paper. Available at http://ssrn.com/abstract=1644530.

Sunstein, C. R. (2009), A constitution of many minds: why the founding document doesn't mean what it meant before, Princeton University Press, Princeton, N.J.

Spirtes, P.; Glymour, C. \& Scheines, R. (1993), Causation, prediction, and search, Springer, New York.

Surowiecki, J. (2004), The wisdom of crowds: why the many are smarter than the few and how collective wisdom shapes business, economies, societies, and nations, Little Brown, London.

Vermeule, A. (2009), 'Many Minds Arguments in Legal Theory', Journal of Legal Analysis 1(1), 1-45.

\section{A The causal foundations of New Independence}

We here introduce causal network terminology more precisely, and give sufficient conditions on causal interconnections for New Independence, citing a result to be proved in a more technical follow-up paper. In this appendix, causal relations are part of the formal setup, and the problem $\boldsymbol{\pi}$ is not a primitive but is defined as the complex random variable consisting of all common causes of votes plus the state $\mathbf{x}$ 
(if not already a common cause). In this setup, we give a formal justification of New Independence in the form of a theorem which derives, rather than assumes, New Independence, based on plausible conditions on the causal relations, which are met in figures 1-3 and in many other causal networks.

In a causal network, a variable $\mathbf{a}$ is said to be a direct cause of another $\mathbf{b}$ (and $\mathbf{b}$ a direct effect of $\mathbf{a}$ ) if there is an arrow pointing from $\mathbf{a}$ to $\mathbf{b}(' \mathbf{a} \rightarrow \mathbf{b}$ '). Further, $\mathbf{a}$ is a cause of $\mathbf{b}$ (and $\mathbf{b}$ an effect of $\mathbf{a}$ ) if there is a directed path from $\mathbf{a}$ to $\mathbf{b}$, i.e., a sequence of variables starting with $\mathbf{a}$ and ending with $\mathbf{b}$ such that each of these variables (except from the last one) directly causes the next one. A variable is a common cause (effect) of some variables if it is a cause (effect) of each of them. A variable is a private cause of a vote if it is a cause of this vote but of no other votes.

The following can be proved:

Theorem (informally stated). Suppose the votes $\mathbf{v}_{1}, \mathbf{v}_{2}, \ldots$ and the state $\mathbf{x}$ are part of a causal network over these and any number of other random variables, and suppose probabilities are compatible with these causal relations. ${ }^{18}$

(a) If no vote is a cause of any other vote, then the votes are independent conditional on the set $\mathcal{C}$ of all common causes of some votes;

(b) If no vote is a cause of any other vote, and the state $\mathbf{x}$ is not a common effect of any votes or private causes thereof ${ }^{19}$, then the votes are also independent conditional on the augmented set $\mathcal{C} \cup\{\mathbf{x}\}$ (i.e., New Independence holds with the problem identified with this augmented set).

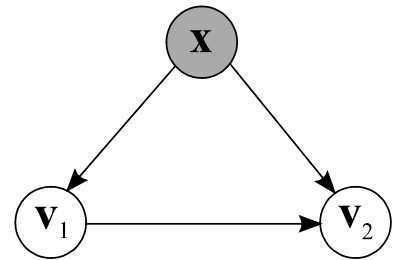

(a)

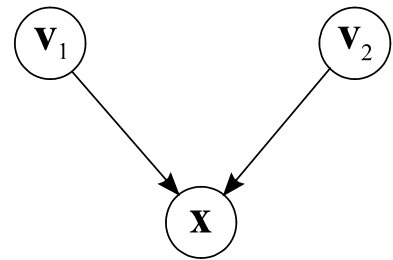

(b)

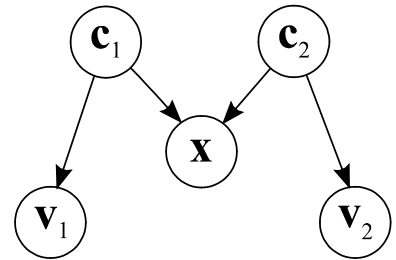

(c)

Figure 6: Violations of New Independence.

Many different causal networks, such as those in figures 1-3, meet the conditions laid out in part (b) of the theorem. In such networks the votes are independent conditional on the problem and New Independence holds. However, some rather special causal setups violate the conditions of part (b), hence of New Independence. We give three elementary examples in figure 6 . Note that votes have no common causes in $6 \mathrm{~b}$ and $6 \mathrm{c}$, and have just $\mathbf{x}$ as a common cause in $6 \mathrm{a}$, so that in all three cases the problem on which we conditionalize consists of $\mathbf{x}$ alone. Therefore, New and Classical Independence here reduce to the same condition, so that the figures

\footnotetext{
${ }^{18}$ Compatibility means that any variable is independent of its non-effects conditional on its direct causes. This requirement is called the Parental Markov Condition.

${ }^{19} \mathrm{In}$ other words, the state $\mathbf{x}$ is not a common effect of variables each of which is or privately causes a different vote.
} 
are in fact counterexamples to both independence conditions. In 6a, vote 1 influences vote 2 . With such a direct causal connection between the votes independence is clearly violated. In $6 \mathrm{~b}$, the votes cause the state of the world, which is implausible. Such a network could arise if the state of the world (the correct judgment) was simply defined as the majority judgment, but this is incompatible with a procedureindependent notion of correctness as usually assumed in epistemic conceptions of democracy. Again, independence is violated. ${ }^{20}$

A more complicated case is displayed in figure 6c. Here the state is the causal product of two different private causes of votes. Independence would require that the votes are independent conditional on the state. But this is not the case, as we can see by considering the following interpretation of causal network 6c. Suppose state $\mathbf{x}$ represents the fact of whether parties A and B will form a coalition. Suppose, for simplicity, that A and B will form a coalition if and only if in each party a majority of members supports forming the coalition. $\mathbf{c}_{1}$ represents the fact of whether there is enough support in A, $\mathbf{c}_{2}$ of whether there is enough support in B. Voter 1 is an expert for party A, and bases his prediction entirely on the mood in party A (thus $\mathbf{c}_{1}$ 's causal effect on $\mathbf{v}_{1}$ ), while voter 2 is an expert for party $B$ and bases her prediction only on the mood in B (thus $\mathbf{c}_{2}$ 's causal effect on $\mathbf{v}_{2}$ ). Thus, each voter votes that the coalition takes place just in case he or she thinks there is enough support in the party studied. Imagine we know that the state is, say, that the coalition does not take place. If Independence were true, then voter 1's vote could not tell us anything about voter 2's vote. But voter 1's vote can tell us something: if voter 1 votes, say, for the coalition taking place, then voter 2 probably votes the opposite, because voter 1 's vote indicates that party $\mathrm{A}$ is willing, and hence (since the coalition does not take place) that party B refuses to coalesce, which voter 2 will know.

The upshot of this discussion is that our New Independence assumption will hold for most causal setups, but is violated when either there is a direct causal relation between the votes, or the state is itself commonly caused by votes or private causes thereof. This is not only a problem for New Independence-Classical Independence is also violated under such circumstances.

\section{B Proof of the New Jury Theorem}

Suppose New Independence and New Competence hold. Two preliminary remarks are due. First, the proof for simplicity assumes that the set of all possible problems, to be denoted $\Pi$, is countable (with each of its subsets being measurable), and that every problem $\pi \in \Pi$ occurs with positive probability: $\operatorname{Pr}(\pi)>0$. As a result, problem-specific competence $p^{\boldsymbol{\pi}}\left(\equiv \operatorname{Pr}\left(R_{i} \mid \boldsymbol{\pi}\right)\right)$ has a discrete distribution over $[0,1]$, supported by a finite set (as in figure 4 ) or a countably infinite set. A proof without

\footnotetext{
${ }^{20}$ If Independence were true, then given that the state is 1 the event of voter 1 voting for 1 would be independent of the event that all other voters vote for 1 . But these two events are negatively correlated: $\operatorname{Pr}\left(\mathbf{v}_{1}=1 \mid \mathbf{v}_{2}=\ldots=\mathbf{v}_{n}=1, \mathbf{x}=1\right)<\operatorname{Pr}\left(\mathbf{v}_{1}=1 \mid \mathbf{x}=1\right)$. The reason for this inequality is that the left hand side reduces to $\operatorname{Pr}\left(\mathbf{v}_{1}=1 \mid \mathbf{v}_{2}=\ldots=\mathbf{v}_{n}=1\right.$ ) (since the event that $\mathbf{v}_{2}=\ldots=\mathbf{v}_{n}=1$ implies that $\mathbf{x}=1$ ), which equals $\operatorname{Pr}\left(\mathbf{v}_{1}=1\right)$ (since the votes are independent unconditionally), which in turn is smaller than $\operatorname{Pr}\left(\mathbf{v}_{1}=1 \mid \mathbf{x}=1\right.$ ) (since the events that $\mathbf{v}_{1}=1$ and that $\mathbf{x}=1$ are positively correlated because 1 's voting for 1 partially explains that $\mathbf{x}=1$ ).
} 
this restriction is available on request; it expresses expectations using integrals instead of sums and relies on the general statement of New Competence mentioned in footnote 14 .

Second, some of the summations used below involve an apparently uncountable number of terms (as in ' $\sum_{p \in[0,1]} \cdots$ '). Nonetheless these sums are well-defined because the number of non-zero terms is always countable (and because if this number is countably infinite then the sum converges to a value that does not depend on the order of summation).

Fix any group size $n$ in $\{1,3,5, \ldots\}$. For any problem $\pi \in \Pi$ consider $\operatorname{Pr}\left(M_{n} \mid \pi\right)$, the conditional probability that the number of correct votes exceeds $\frac{n}{2}$ given the problem $\pi$. Given the problem $\pi$, this number is the sum of $n$ independent Bernoulli variables with parameter $p^{\pi}$ (by New Independence and New Competence). Hence this number follows a Binomial distribution with parameters $n$ and $p^{\pi}$, that is, takes each value $m$ in $\{0,1, \ldots, n\}$ with probability $\frac{n !}{m !(n-m) !}\left(p^{\pi}\right)^{n}\left(1-p^{\pi}\right)^{n-m}$, and more generally falls into each set of values $S \subseteq \mathbb{R}$ with a probability of

$$
B_{n, p^{\pi}}(S):=\sum_{m \in\{0,1, \ldots, n\} \cap S} \frac{n !}{m !(n-m) !}\left(p^{\pi}\right)^{m}\left(1-p^{\pi}\right)^{n-m} .
$$

To obtain the probability of a correct majority given $\pi$, we have to take $S=\left(\frac{n}{2}, n\right]$ :

$$
\operatorname{Pr}\left(M_{n} \mid \pi\right)=B_{n, p^{\pi}}\left(\left(\frac{n}{2}, n\right]\right) .
$$

By averaging over all possible problems, we obtain the unconditional probability that a majority is correct:

$$
\operatorname{Pr}\left(M_{n}\right)=\sum_{\pi \in \Pi} \operatorname{Pr}\left(M_{n} \mid \pi\right) \operatorname{Pr}(\pi)=\sum_{\pi \in \Pi} B_{n, p^{\pi}}\left(\left(\frac{n}{2}, n\right]\right) \operatorname{Pr}(\pi) .
$$

By partitioning the set of all problems $\Pi$ into subsets of problems with equal voter competence $p \in[0,1]$, the summation ' $\sum_{\pi \in \Pi}$ ' becomes equivalent to a nested summation ' $\sum_{p \in[0,1]} \sum_{\pi \in \Pi: p^{\pi}=p}$ '. Thereby we obtain that

$$
\begin{aligned}
\operatorname{Pr}\left(M_{n}\right) & =\sum_{p \in[0,1]} B_{n, p}\left(\left(\frac{n}{2}, n\right]\right) \sum_{\pi \in \Pi: p^{\pi}=p} \operatorname{Pr}(\pi) \\
& =\sum_{p \in[0,1]} B_{n, p}\left(\left(\frac{n}{2}, n\right]\right) \operatorname{Pr}\left(p^{\pi}=p\right) .
\end{aligned}
$$

We split the last expression into a sum $S_{1}+S_{2}+S_{3}$, where $S_{1}, S_{2}$ and $S_{3}$ are defined by restricting the summation index $p$ to values above, below, or equal to $\frac{1}{2}$, respectively. Specifically, $S_{1}$ is given by

$$
S_{1}=\sum_{p \in\left(\frac{1}{2}, 1\right]} B_{n, p}\left(\left(\frac{n}{2}, n\right]\right) \operatorname{Pr}\left(p^{\boldsymbol{\pi}}=p\right) .
$$


Next, $S_{2}$ is given by

$$
\begin{aligned}
S_{2} & =\sum_{p \in\left[0, \frac{1}{2}\right)} B_{n, p}\left(\left(\frac{n}{2}, n\right]\right) \operatorname{Pr}\left(p^{\boldsymbol{\pi}}=p\right) \\
& =\sum_{p \in\left[0, \frac{1}{2}\right)}\left\{1-B_{n, p}\left(\left[0, \frac{n}{2}\right)\right)\right\} \operatorname{Pr}\left(p^{\boldsymbol{\pi}}=p\right) \\
& =\sum_{p \in\left[0, \frac{1}{2}\right)} \operatorname{Pr}\left(p^{\boldsymbol{\pi}}=p\right)-\sum_{p \in\left[0, \frac{1}{2}\right)} B_{n, p}\left(\left[0, \frac{n}{2}\right)\right) \operatorname{Pr}\left(p^{\boldsymbol{\pi}}=p\right) .
\end{aligned}
$$

This expression is the difference of (i) a sum which reduces to $\operatorname{Pr}\left(p^{\pi}<\frac{1}{2}\right)$, and (ii) another sum which (through a change of variable from $p$ to $1-p$ ) can be rewritten as $\sum_{p \in\left(\frac{1}{2}, 1\right]} B_{n, 1-p}\left(\left[0, \frac{n}{2}\right)\right) \operatorname{Pr}\left(p^{\pi}=1-p\right)$. Here, $B_{n, 1-p}\left(\left[0, \frac{n}{2}\right)\right)$ can in turn be rewritten as $B_{n, p}\left(\left(\frac{n}{2}, n\right]\right)$; the reason is that

$$
\begin{aligned}
B_{n, 1-p}\left(\left[0, \frac{n}{2}\right)\right) & =\sum_{m=0, \ldots, \frac{n-1}{2}} \frac{n !}{m !(n-m) !}(1-p)^{m} p^{n-m} \text { and } \\
B_{n, p}\left(\left(\frac{n}{2}, n\right]\right) & =\sum_{m=\frac{n+1}{2}, \ldots, n} \frac{n !}{m !(n-m) !} p^{m}(1-p)^{n-m},
\end{aligned}
$$

where the sums appearing on the right hand sides coincide, as is seen from a change of variable from $m$ to $n-m$. We have thus shown that

$$
S_{2}=\operatorname{Pr}\left(p^{\pi}<\frac{1}{2}\right)-\sum_{p \in\left(\frac{1}{2}, 1\right]} B_{n, p}\left(\left(\frac{n}{2}, n\right]\right) \operatorname{Pr}\left(p^{\pi}=1-p\right) .
$$

Finally, $S_{3}$ is given by

$$
S_{3}=B_{n, \frac{1}{2}}\left(\left(\frac{n}{2}, n\right]\right) \operatorname{Pr}\left(p^{\pi}=\frac{1}{2}\right) .
$$

Here, $B_{n, \frac{1}{2}}\left(\left(\frac{n}{2}, n\right]\right)$ is, just as $B\left(\left[0, \frac{n}{2}\right)\right)$, equal to $\frac{1}{2}$ because the binomial distribution $B_{n, \frac{1}{2}}$ is symmetric around its mean $\frac{n}{2}$. So,

$$
S_{3}=\frac{1}{2} \operatorname{Pr}\left(p^{\pi}=\frac{1}{2}\right) .
$$

By adding the expressions 1,2 and 3, we obtain that

$$
\operatorname{Pr}\left(M_{n}\right)=S_{1}+S_{2}+S_{3}=\sum_{p \in\left(\frac{1}{2}, 1\right]} B_{n, p}\left(\left(\frac{n}{2}, n\right]\right) \Delta(p)+\Gamma,
$$

where $\Delta(p)$ and $\Gamma$ are defined as follows:

$$
\begin{aligned}
\Delta(p) & =\operatorname{Pr}\left(p^{\boldsymbol{\pi}}=p\right)-\operatorname{Pr}\left(p^{\boldsymbol{\pi}}=1-p\right), \\
\Gamma & =\operatorname{Pr}\left(p^{\boldsymbol{\pi}}<\frac{1}{2}\right)+\frac{1}{2} \operatorname{Pr}\left(p^{\boldsymbol{\pi}}=\frac{1}{2}\right) .
\end{aligned}
$$


Regarding our theorem's non-asymptotic conclusion, we have to show that the expression 4 is increasing in the odd group size $n$. Since $\Gamma$ is a constant in $n$ and since $\Delta(p)$ is a non-negative constant by New Competence, it suffices to show that for fixed $p \in\left(\frac{1}{2}, 1\right]$ the binomial probability

$$
P_{n}:=B_{n, p}\left(\left(\frac{n}{2}, n\right]\right)
$$

is increasing in (odd) $n$. This follows from a recursion formula:

$$
P_{n+2}=P_{n}+(2 p-1)\left(\begin{array}{c}
n \\
\frac{n+1}{2}
\end{array}\right)[p(1-p)]^{\frac{n+1}{2}} .
$$

While this recursion formula appears in the literature (e.g., Grofman et. al 1983), we cannot find a published derivation; let us therefore mention a simple combinatorial argument to its effect. We again conditionalize on a given problem $\pi \in \Pi$ and write $p$ for the corresponding problem-specific competence $p^{\pi}$. Fix any odd group size $n$ $(\in\{1,3, \ldots\})$. Recall that $P_{n+2}$ is the (problem-conditional) probability of the event $M_{n+2}$ that more than half of the first $n+2$ votes is correct. This event can be partitioned into two subevents: the subevent $M_{n+2} \backslash M_{n}$ that more than half of the first $n+2$ but fewer than half of the first $n$ votes are correct, and the subevent $M_{n+2} \cap M_{n}$ that more than half of the first $n+2$ and also more than half of the first $n$ votes are correct. We can therefore decompose $P_{n+2}$ into a sum:

$$
P_{n+2}=\operatorname{Pr}\left(M_{n+2} \backslash M_{n} \mid \pi\right)+\operatorname{Pr}\left(M_{n+2} \cap M_{n} \mid \pi\right) .
$$

Here, the second term can in turn be rewritten as follows:

$$
\operatorname{Pr}\left(M_{n+2} \cap M_{n} \mid \pi\right)=\operatorname{Pr}\left(M_{n} \mid \pi\right)-\operatorname{Pr}\left(M_{n} \backslash M_{n+2} \mid \pi\right)=P_{n}-\operatorname{Pr}\left(M_{n} \backslash M_{n+2} \mid \pi\right),
$$

so that in summary

$$
P_{n+2}=P_{n}+\operatorname{Pr}\left(M_{n+2} \backslash M_{n} \mid \pi\right)-\operatorname{Pr}\left(M_{n} \backslash M_{n+2} \mid \pi\right) .
$$

Now the event $M_{n+2} \backslash M_{n}$ implies that exactly $\frac{n+1}{2}$ of the first $n$ votes (i.e., a narrow majority) must be incorrect - which happens with probability $\left(\begin{array}{c}n+1 \\ \frac{n+1}{2}\end{array}\right) p^{\frac{n-1}{2}}(1-p)^{\frac{n+1}{2}}$ while the next two votes must be correct-which happens with probability $p^{2}$. By multiplication we therefore obtain that

$$
\begin{aligned}
\operatorname{Pr}\left(M_{n+2} \backslash M_{n} \mid \pi\right) & =\left(\begin{array}{c}
n \\
\frac{n+1}{2}
\end{array}\right) p^{\frac{n-1}{2}}(1-p)^{\frac{n+1}{2}} p^{2} \\
& =p\left(\begin{array}{c}
n \\
\frac{n+1}{2}
\end{array}\right)[p(1-p)]^{\frac{n+1}{2}} .
\end{aligned}
$$

Similarly, the event $M_{n} \backslash M_{n+2}$ of a correct majority among the first $n$ but not among the first $n+2$ votes implies that exactly $\frac{n+1}{2}$ of the first $n$ votes (i.e., a narrow majority) is correct - which has probability $\left(\begin{array}{c}n \\ \frac{n+1}{2}\end{array}\right) p^{\frac{n+1}{2}}(1-p)^{\frac{n-1}{2}}$ - while the next two 
votes are incorrect-which has probability $(1-p)^{2}$. Thus, again by multiplication,

$$
\begin{aligned}
\operatorname{Pr}\left(M_{n} \backslash M_{n+2} \mid \pi\right) & =\left(\begin{array}{c}
n \\
\frac{n+1}{2}
\end{array}\right) p^{\frac{n+1}{2}}(1-p)^{\frac{n-1}{2}}(1-p)^{2} \\
& =(1-p)\left(\begin{array}{c}
n \\
\frac{n+1}{2}
\end{array}\right)[p(1-p)]^{\frac{n+1}{2}} .
\end{aligned}
$$

Using the expressions 7 and 8, the equation 6 implies the recursion formula 5 .

As for the asymptotic conclusion, it suffices to note that as $n \rightarrow \infty$ the binomial probability $P_{n}$ converges to one, so that by 4 we have

$$
\begin{aligned}
\operatorname{Pr}\left(M_{n}\right) & \rightarrow \sum_{p \in\left(\frac{1}{2}, 1\right]} \Delta(p)+\Gamma \\
& =\sum_{p \in\left(\frac{1}{2}, 1\right]} \operatorname{Pr}\left(p^{\boldsymbol{\pi}}=p\right)-\sum_{p \in\left(\frac{1}{2}, 1\right]} \operatorname{Pr}\left(p^{\boldsymbol{\pi}}=1-p\right)+\Gamma \\
& =\operatorname{Pr}\left(p^{\boldsymbol{\pi}}>\frac{1}{2}\right)-\operatorname{Pr}\left(p^{\boldsymbol{\pi}}>\frac{1}{2}\right)+\Gamma \\
& =\operatorname{Pr}\left(p^{\boldsymbol{\pi}}>\frac{1}{2}\right)+\frac{1}{2} \operatorname{Pr}\left(p^{\boldsymbol{\pi}}=\frac{1}{2}\right) .
\end{aligned}
$$

This limit is one if $\operatorname{Pr}\left(p^{\pi}>\frac{1}{2}\right)=1$, and less than one otherwise. 